\title{
Autonomic, Cardiovascular and Respiratory Responses to Hyperglycemic Stimulus in Healthy Subjects
}

\author{
Salvador Carrasco-Sosa, Alejandra Guillén-Mandujano
}

\author{
División Ciencias Biológicas y de la Salud, Universidad Autónoma Metropolitana-I, CDMX, México
}

\begin{abstract}
In ten healthy subjects we assessed the effects of hyperglycemia, provoked by the ingestion of $75 \mathrm{~g}$ of glucose on: $R-R$ intervals (RR), systolic pressure (SP), diastolic pressure $(D P)$, respiratory frequency $(R F)$ and tidal volume $\left(V_{T}\right)$ 5-min time series; the time course of their low-frequency $\left(L F_{R R}, L F_{S P}, L F_{D P}\right)$, high-frequency $\left(H F_{R R}, H F_{D P}, H F_{R e s}\right)$ powers and their central frequencies $\left(c f L F_{D P}, c f H F_{R R}, c f H F_{D P}\right)$, computed by a time-frequency distribution; baroreflex (BRS) and respiratory sinus arrhythmia sensitivities (RSAS), obtained by alpha index and their coherences (cBRS and cRSAS) by cross timefrequency analysis. In relation to control, in hyperglycemia (peak of $143 \pm 12 \mathrm{mg} / 100 \mathrm{ml}, \mathrm{p}<0.001$ ) 1 min epoch mean values of: $L F_{R R}, L F_{D P}, H F_{R R}, H F_{R e s}$, $B R S, c B R S, R R, D P$ and $V_{T}$ decreased ( $\left.p<0.03\right) ; c f L F_{D P}$, $c f H F_{R R}, R S A S, c R S A S$ and $R F$ increased ( $\left.p<0.04\right)$; and $L F_{S P}$ and $S P$ were similar. Our findings outline an integrative dynamic response to hyperglycemia characterized by: vagal activity inhibition associated to RR shortening; sympathetic outflow inhibition associated to reduced DP, which, via baroreflex with reduced sensitivity and input-output coupling degree, reinforces the vagal reduction; and respiratory activity modification associated to $V_{T}$ decrease, $R F$ increase and improved respiratory modulation of cardiovascular function.
\end{abstract}

\section{Introduction}

Although hyperglycemia is a common condition presented postprandially in healthy subjects and chronically sustained in diabetic patients, its autonomic, cardiovascular and respiratory (ACR) effects are not clearly understood $[1,2]$, partially because they have been poorly studied and the reported findings in healthy subjects are equivocal. For instance, studies evaluating spectral measures of cardiovascular variability (CVV), have reported that sympathetic activity increases [1] or remains unchanged [2], and that vagal activity either increases [3], does not change [1,2] or decreases [4]. In diabetic patients, the postprandial fluctuations of hyperglycemia evoke depression of sympathetic and vagal activities, additionally influenced by the presence or lack of autonomic neuropathy [5]. To provide some insight into this issue, by using a robust methodology that comprises the analysis of time series of cardiovascularrespiratory variables and of time-frequency measures of vagal and sympathetic activities and autonomic control, we sought to characterize an integrative response to hyperglycemia in healthy individuals. Thus, our aim was to assess the effects of hyperglycemia on heart rate (HR), arterial pressure $(\mathrm{AP})$, tidal volume $\left(\mathrm{V}_{\mathrm{T}}\right)$ and respiratory frequency $(\mathrm{RF})$, the power and central frequencies of spectral measures of HRV and AP variability, baroreflex (BRS) and respiratory sinus arrhythmia sensitivities (RSAS). Correlations among indexes were examined.

\section{Methodology}

\subsection{Subjects}

Ten euglycemic, normotensive and sedentary subjects, 7 men and 3 women, were studied. Their mean age, height and weight were $22.6 \pm 2.0$ years, $165 \pm 7 \mathrm{~cm}$ and $64 \pm 6 \mathrm{~kg}$ respectively. Their written informed consent was requested to participate.

\subsection{Protocol}

Volunteers visited the laboratory twice. The first time, their health status and anthropometric variables were evaluated, and on the second visit the experimental stage was carried out. After an overnight fast, subjects drank 75 gr of glucose. A blood sample of $35 \mu$ l was collected from a fingertip every 10 minutes for the following 180 min to measure blood glucose level by enzymatic method (Boehringer). In supine position, 5-min signal recordings were obtained before glucose ingestion (control), $40 \mathrm{~min}$ after, during the hyperglycemic peak, and when glucose returned to baseline (recovery), around 180 min later.

\subsection{Signal recording and acquisition}

ECG was detected at the CM5 bipolar lead using a 
bioelectric amplifier (Biopac Systems). Noninvasive AP was measured by Finapres (Ohmeda). Respiration (Res) was detected by a pneumograph (Nihon Kohden). All recorded signals were digitized at a sampling rate of 1 $\mathrm{kHz}$ via an acquisition system (Biopac Systems).

\subsection{Data processing}

Maxima and minima of ECG, AP and Res signals were detected to generate time series of R-R intervals (RR), systolic pressure (SP), diastolic pressure (DP), $\mathrm{V}_{\mathrm{T}}$ and RF. All series were cubic-spline interpolated, detrended and resampled at $4 \mathrm{~Hz}$. Time-frequency spectra of the series were estimated with the smoothed pseudo-WignerVille distribution and integrated in the standard frequency bands of HRV analysis to compute their instantaneous low frequency powers $\left(\mathrm{LF}_{\mathrm{RR}}, \mathrm{LF}_{\mathrm{SP}}\right.$ and $\left.\mathrm{LF}_{\mathrm{DP}}\right)$, high frequency powers $\left(\mathrm{HF}_{\mathrm{RR}}, \mathrm{HF}_{\mathrm{DP}}\right.$ and $\left.\mathrm{HF}_{\mathrm{Res}}\right)$ and their central frequencies $\left(\mathrm{cfLF}_{\mathrm{DP}}, \mathrm{cfHF}_{\mathrm{RR}}\right.$ and $\left.\mathrm{cfHF}_{\mathrm{DP}}\right)$. Instantaneous BRS and RSAS were computed by alpha index, using $\mathrm{LF}_{\mathrm{RR}} / \mathrm{LF}_{\mathrm{SP}}$ and $\mathrm{HF}_{\mathrm{RR}} / \mathrm{HF}_{\mathrm{Res}}$ respectively, and their coherences (cBRS and cRSAS) were estimated by cross time-frequency analysis. Measures dynamics were expressed as change from their mean baseline, segmented into 1-min epochs for statistical analysis and ensembleaveraged for visualization.

\subsection{Statistical analysis}

Data of the measures were expressed as mean $\pm \mathrm{SD}$. Differences between baseline, hyperglycemia and recovery values were tested by ANOVA for repeated measures. Post-hoc pairwise comparisons were performed by the Tukey test. Using the 1-min epochs means, linear regressions and correlations between the indexes were computed for each subject. Statistical significance was accepted at $\mathrm{p}<0.05$.

\section{Results}

In relation to glycemia control level $(82 \pm 10 \mathrm{mg} / 100$ $\mathrm{ml}$ ), around $40 \mathrm{~min}$ after glucose ingestion a hyperglycemic peak of $143 \pm 12 \mathrm{mg} / 100 \mathrm{ml}(\mathrm{p}<0.001)$ was reached. Glycemia returned to baseline after $120 \mathrm{~min}$.

Relative to control (Fig. 1A and C), time-frequency spectra of RR and DP series during hyperglycemia showed reductions of $\mathrm{HF}_{\mathrm{RR}}$ and $\mathrm{LF}_{\mathrm{RR}}$ powers (with two overshoots) with increase of $\mathrm{cfHF}_{\mathrm{RR}}$ (Fig. 1B), and $\mathrm{LF}_{\mathrm{DP}}$ reduction (with an overshoot) and $\mathrm{cfLF}_{\mathrm{DP}}$ increment (Fig. 1D). Instantaneous powers and central frequencies presented strikingly large fluctuations during baseline and hyperglycemia.

In relation to baseline, in hyperglycemia 1-min epoch mean values of instantaneous response patterns of the three types of measures behaved as follows:

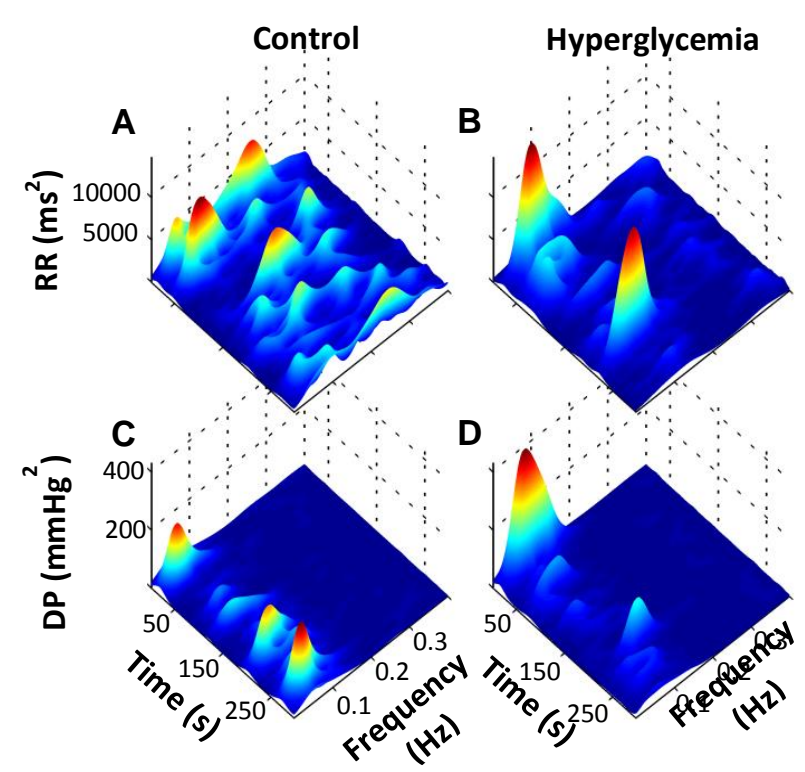

Fig. 1. Representative time-frequency distributions of RR and DP series during control (A and $\mathrm{C}$ ) and hyperglycemia (B and $\mathrm{D})$ respectively.

- Cardiovascular and respiratory function: RR (Fig. 2A), DP (except one epoch mean, Fig. 2B), and $\mathrm{V}_{\mathrm{T}}$ (Fig. 2C), were smaller ( $<<0.04)$; RF (Fig. 2D) was larger $(\mathrm{p}<0.03)$; and SP was similar. In recovery, RF was greater $(\mathrm{p}<0.04)$ and $\mathrm{V}_{\mathrm{T}}$ was smaller $(\mathrm{p}<0.02)$ than their control.

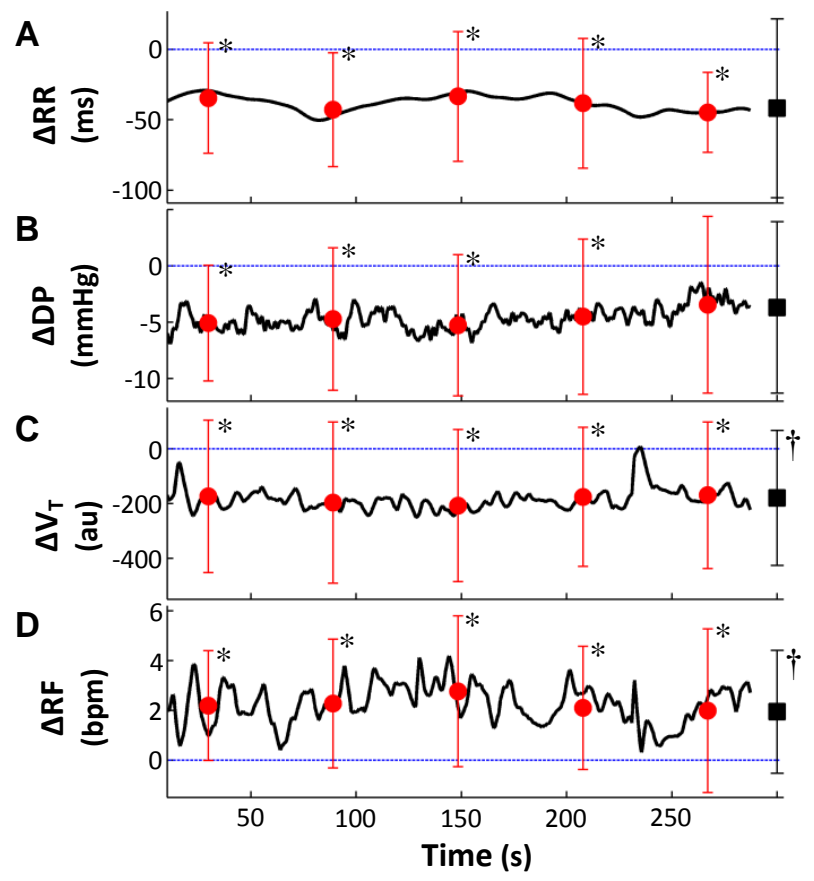

Fig. 2. Ensemble averages and 1-min epoch means \pm SD of the dynamics of cardiovascular respiratory variables: A)RR, B)DP, C) $V_{\mathrm{T}}$ and D)RF. $\mathbf{m}=$ mean recovery. ${ }^{*} \mathrm{p}<0.04$ hyperglycemia vs. control. $\uparrow \mathrm{p}<0.04$ recovery vs. control. 
- Autonomic activity: $\mathrm{LF}_{\mathrm{RR}}$ (Fig. $3 \mathrm{~A}$ ), $\mathrm{LF}_{\mathrm{DP}}$ (except two epoch means, one was greater and the other similar, Fig. 3C), $\operatorname{lnHF}_{\mathrm{RR}}$ (Fig. 3D), $\mathrm{HF}_{\text {Res }}$ (Fig. 3E) and $\mathrm{HF}_{\mathrm{DP}}$ were smaller $(p<0.04)$; $c f L F_{D P}$ (except one epoch mean, Fig. $3 \mathrm{~F}), \mathrm{cfHF}_{\mathrm{DP}}(\mathrm{Fig} .3 \mathrm{G})$ and $\mathrm{cfHF}_{\mathrm{RR}}$ were larger $(\mathrm{p}<0.04)$; and $\mathrm{LF}_{\mathrm{SP}}$ was similar (Fig. $3 \mathrm{~B}$ ). In recovery, $\mathrm{HF}_{\mathrm{Res}}$ was less than its baseline $(\mathrm{p}<0.01)$.

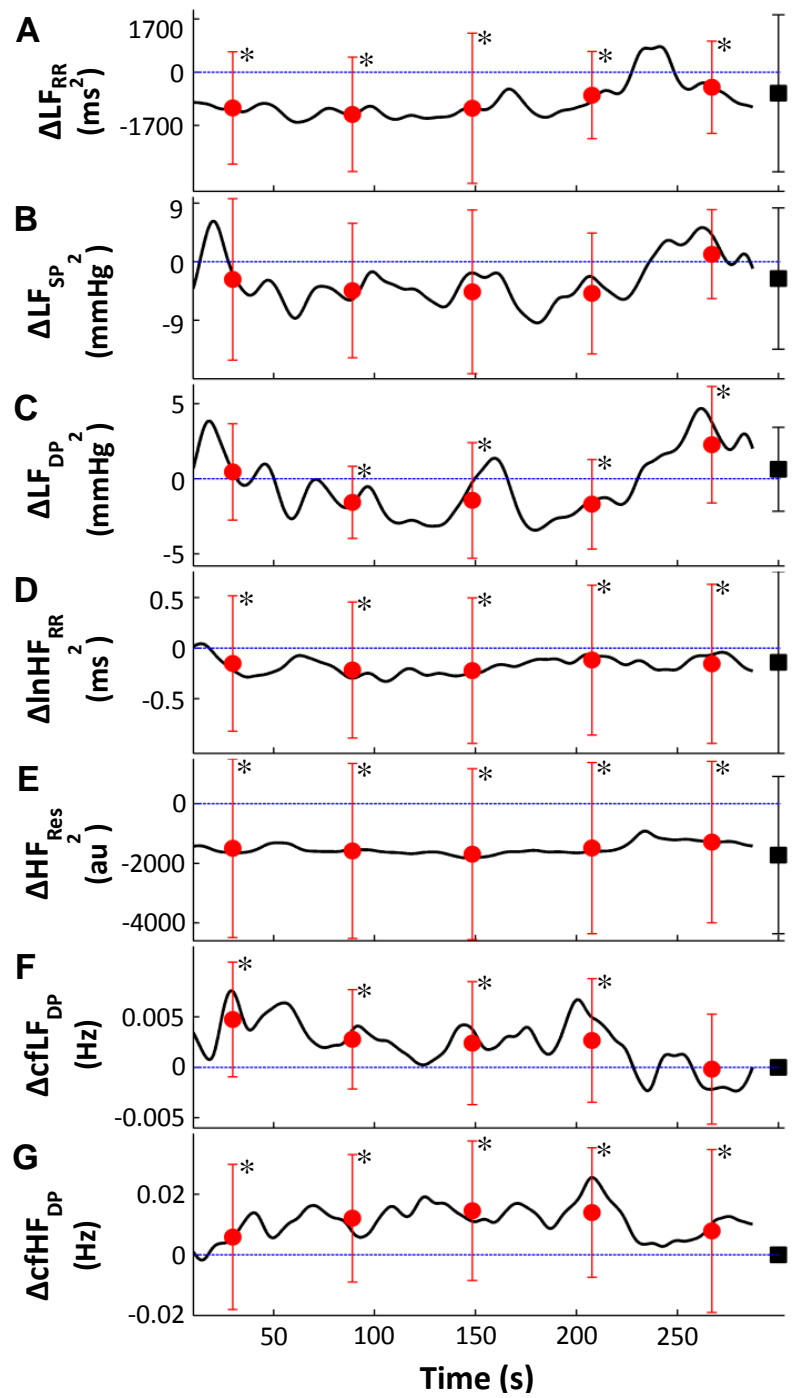

Fig. 3. Ensemble averages and 1-min epoch means \pm SD of the dynamics of spectral autonomic activity measures: A) $\left.\left.\left.\left.\left.\mathrm{LF}_{\mathrm{RR}}, \mathrm{B}\right) \mathrm{LF}_{\mathrm{SP}}, \mathrm{C}\right) \mathrm{LF}_{\mathrm{DP}}, \mathrm{D}\right) \operatorname{lnHF}_{\mathrm{RR}}, \mathrm{E}\right) \mathrm{HF}_{\mathrm{Res}}, \mathrm{F}\right) \mathrm{cfLF}_{\mathrm{DP}}$ and G) $\quad \mathrm{cfHF}_{\mathrm{DP}}$. =mean recovery. ${ }^{*} \mathrm{p}<0.04$ hyperglycemia vs. control. $\uparrow \mathrm{p}<0.01$ recovery vs. control

- Autonomic control mechanisms: BRS (Fig. 4A) and cBRS (except two epoch means, Fig. 4B) were smaller $(\mathrm{p}<0.04)$; RSAS (Fig. 4C) and cRSAS (except one epoch mean, Fig. 4D) were greater $(\mathrm{p}<0.02)$. Control mean value of cBRS was $0.77 \pm 0.09$ and that of cRSAS was $0.92 \pm 0.05$. In recovery, cRSAS was greater than its value in control $(\mathrm{p}<0.02)$.

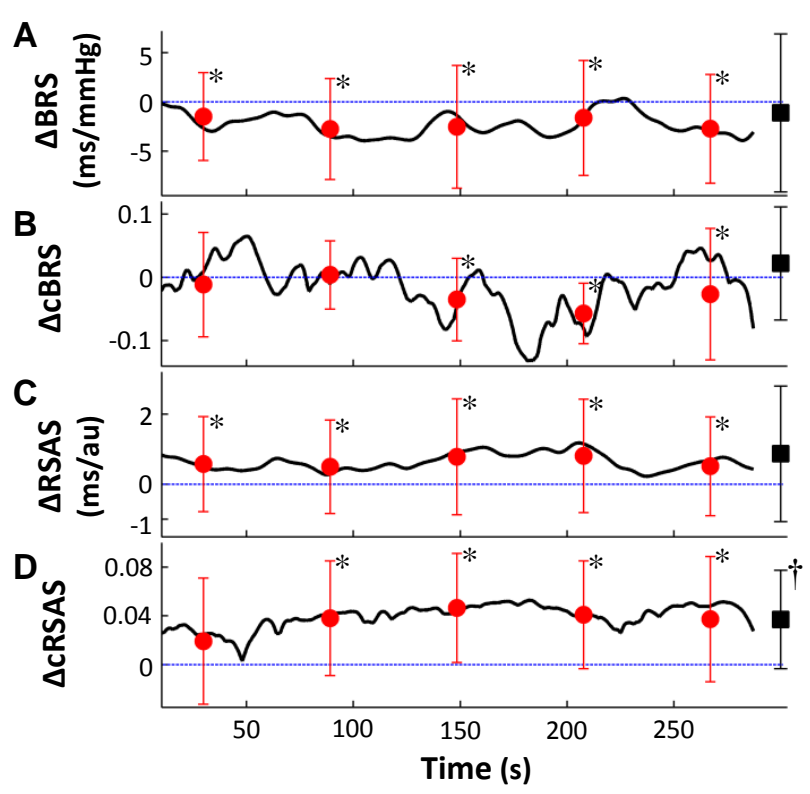

Fig. 4. Ensemble averages and 1-min epoch means \pm SD of the dynamics of autonomic control estimators: A)BRS, B)cBRS, C)RSAS, D)cRSAS. m=mean recovery. $* \mathrm{p}<0.04$ hyperglycemia vs. control. $\uparrow \mathrm{p}<0.02$ recovery vs. control

$\mathrm{cfLF}_{\mathrm{DP}}-\mathrm{LF}_{\mathrm{DP}}$ relation was inverse (Fig. 5A) and $\mathrm{cfHF}_{\mathrm{DP}}$-RF (Fig. 5B), BRS-cBRS (Fig. 5C) and RSAScRSAS (Fig. 5D) relations were direct. $\mathrm{cfHF}_{\mathrm{RR}}-\mathrm{RF}$ correlation was $0.65 \pm 0.12 ; \quad \mathrm{HF}_{\mathrm{RR}}-\mathrm{V}_{\mathrm{T}}$ and $\mathrm{HF}_{\mathrm{DP}}-\mathrm{V}_{\mathrm{T}}$ correlations were $0.36 \pm 0.24$ and $0.28 \pm 0.33$ respectively. All correlations were significant $(\mathrm{p}<0.004)$.
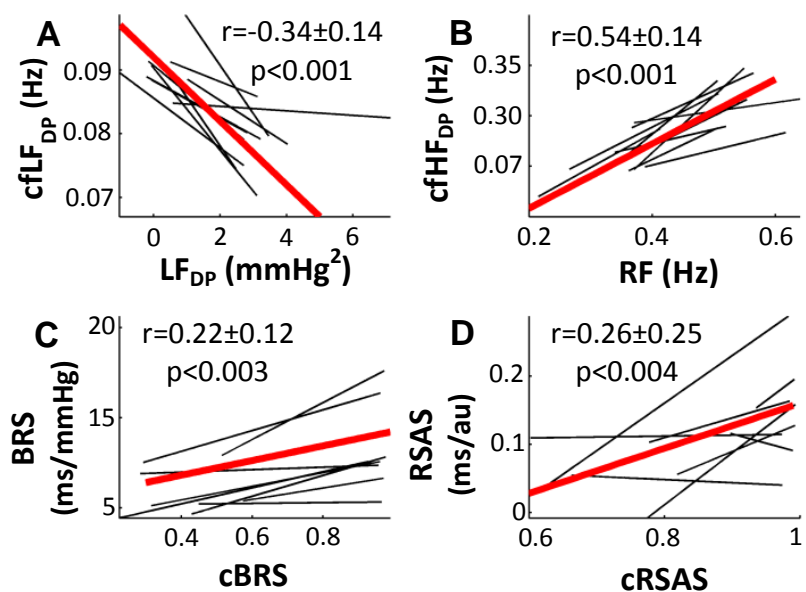

Fig. 5. Individual (thin black lines) and mean (thick red lines) regressions and correlations for: $\mathrm{A}) \mathrm{cfLF}_{\mathrm{DP}}-\mathrm{LF}_{\mathrm{DP}}, \mathrm{B}$ ) $\left.\left.\mathrm{cfHF}_{\mathrm{DP}}-\mathrm{RF}, \mathrm{C}\right) \mathrm{BRS}-\mathrm{cBRS}, \mathrm{D}\right) \mathrm{RSAS}-\mathrm{cRSAS}$ relations.

\section{Discussion}

Our robust analysis methodology revealed an integrative functional picture of the ACR response of healthy subjects to hyperglycemia, supported by our main 
findings in three types of estimators: Cardiovascularrespiratory function, reduction of $\mathrm{DP}, \mathrm{RR}$ and $\mathrm{V}_{\mathrm{T}}$ and increase of RF; sympathetic and vagal activities, reductions of $\mathrm{LF}_{\mathrm{RR}}, \mathrm{LF}_{\mathrm{DP}}, \ln \mathrm{HF}_{\mathrm{RR}}, \mathrm{HF}_{\mathrm{DP}}$ and $\mathrm{HF}_{\mathrm{Res}}$, and increases of cfLF $\mathrm{DP}_{\mathrm{DP}}, \mathrm{cfHF}_{\mathrm{RR}}$ and $\mathrm{cfHF}_{\mathrm{DP}}$; autonomic control mechanisms, decrease of BRS and cBRS and increase of RSAS and cRSAS. Additionally, some degree of covariation is found in $\mathrm{cfLF}_{\mathrm{DP}}-\mathrm{LF}_{\mathrm{DP}}$, BRS-cBRS, RSAS-cRSAS, $\quad \mathrm{HF}_{\mathrm{RR}}-\mathrm{V}_{\mathrm{T}}, \quad \mathrm{HF}_{\mathrm{DP}}-\mathrm{V}_{\mathrm{T}}, \quad \mathrm{cfHF}_{\mathrm{RR}}-\mathrm{RF}$ and $\mathrm{cfHF}_{\mathrm{DP}}-\mathrm{RF}$.

Our methodological approach, based on the use of time series and a time-frequency distribution, a valuable tool for non-stationary signal spectral analysis that avoids the need for stationarity testing, shows that the time course of ACR function measures in hyperglycemia are highly fluctuating, especially the sympathetic ones. To achieve a robust estimation of sympathetic activity we employed three of its most common spectral measures, $\mathrm{LF}_{\mathrm{RR}}, \mathrm{LF}_{\mathrm{SP}}$ and $\mathrm{LF}_{\mathrm{DP}}$, of which $\mathrm{LF}_{\mathrm{SP}}$ (Fig. 3B) fails to mark the effect of hyperglycemia. Additionally, $\mathrm{LF}_{\mathrm{DP}}$ presents weak yet significant negative correlation with $\operatorname{cfLF}_{\mathrm{DP}}$ (Fig. 5A), which suggests that the responses of its power and central frequency to sympathetic activity changes are inversely proportional. Significant correlations of BRS-cBRS (Fig. 5C) and RSAS-cRSAS (Fig. 5D) relations suggest some functional interdependency between the sensitivity of each control system and its coherence, which we consider indicates their degree of input-output coupling. The weak correlations found for the RF-cfHF $\mathrm{RR}_{\mathrm{R}}$, RF-cfHF $\mathrm{DP}_{\mathrm{DP}}$ (Fig. 5B), $\mathrm{V}_{\mathrm{T}}-\mathrm{HF}_{\mathrm{RR}}$ and $\mathrm{V}_{\mathrm{T}}-\mathrm{HF}_{\mathrm{DP}}$ relations, add supporting evidence that the respiratory variables are relevant functional correlates of the spectral components of CVV in the high frequency band, RF for central frequencies and $\mathrm{V}_{\mathrm{T}}$ for powers.

Previous studies on the ACR response of healthy subjects to hyperglycemia have in common that: hyperglycemia has been provoked by either glucose $[2,3]$ or meal ingestion $[4,6]$; to assess its effects, spectral measures of CVV have been used more frequently than microneurography, always assuming stationary signals, which implies the use of ad hoc spectral analysis techniques [1-4,6]; and have attributed the autonomic effects to insulin $[1,6]$. The following effects of hyperglycemia on ACR measures have been reported: HR increment $[1,3,4,7]$ or no change [2]; AP increase [1,6], no change [2] or decrease [3]; sympathetic activity increase $[1,3,6,8]$ or no change [2]; vagal activity increase [3], decrease [4] or no change [1,2]; and BRS decrease $[1,3,7]$. Therefore, the reported ACR effects of hyperglycemia are equivocal; however, most authors consider the sympathoexcitatory pressor effect to be the most relevant one $[1,6,8]$, notion that contrasts with the dynamic and integrative mechanism supported by our findings, consisting in: vagal activity inhibition (Fig. 3D) associated to RR shortening (Fig. 2A); sympathetic activity inhibition (Fig. 3A, C and F) associated to DP reduction (Fig. 2B), which, by baroreflex with reduced sensitivity (Fig. 4A) and input-output coupling degree (Fig. 4B), somewhat contributes to vagal withdrawal; and respiratory activity modification leading to reduced $\mathrm{V}_{\mathrm{T}}$ (Fig. 2C) and increased RF (Fig. 2D), with greater RSAS (Fig. 4C) and degree of respiratory-cardiovascular coupling (Fig. 4D). The sporadic sympathetic bursts (Fig. 3C) transiently recover DP level (Fig. 2B). With the return of glycemia to its control level, the studied measures recover their control values, except for the respiratory variables: RF remains elevated (Fig. 2C) and $\mathrm{V}_{\mathrm{T}}$ persists reduced (Fig. 2D).

In conclusion, our robust methodology based on the analysis of time series, and their time-frequency spectra, and strengthened by the finding of some covariation between $\mathrm{LF}_{\mathrm{DP}}$ and $\mathrm{cfLF}_{\mathrm{DP}}$ and between the sensitivities and their coherences, documents an integrative dynamic response to hyperglycemia characterized by: depressed vagal, sympathetic (with some activation bursts), BRS, $\mathrm{DP}$ and $\mathrm{V}_{\mathrm{T}}$ levels, and increased HR and RF with a higher degree of coupling of respiratory modulation of CVV.

\section{References}

[1] Cao L, Pilowsky P. Quiet standing after carbohydrate ingestion induces sympathoexcitatory and pressor responses in young healthy males. Auton Neurosci 2014;185:112-9.

[2] van Gurp P, Rongen G, Lenders J, et al. Sustained hyperglycaemia increases muscle blood flow but does not affect sympathetic activity in resting humans. Eur J Appl Physiol 2005;93:648-54.

[3] Brown C, Dulloo AG, Yepuri G, Montani J. Fructose ingestion acutely elevates blood pressure in healthy young humans. Am J Physiol 2008;294:R730-7.

[4] Chang C, Ko C, Lien H, Chou M. Varying postprandial abdominovagal and cardiovagal activity in normal subjects. Neurogastroenterol Motil 2010;22:546-52.

[5] Klimontov V, Myakina N, Tyan N. Heart rate variability is associated with interstitial glucose fluctuations in type 2 diabetic women treated with insulin. Springerplus 2016;5:337.

[6] Young C, Deo S, Chaudhary K, et al. Insulin enhances the gain of arterial baroreflex control of muscle sympathetic nerve activity in humans. J Physiol 2010;588:3593-603.

[7] Madden K, Tedder G, Lockhart C, Meneilly G. Oral glucose tolerance test reduces arterial baroreflex sensitivity in older adults. Can J Physiol Pharmacol 2008;86:71-7.

[8] Paolisso G, Manzella D, Ferrara N, Gambardella A, et al. Glucose ingestion affects cardiac ANS in healthy subjects with different amounts of body fat. Am J Physiol 1997;273:E471-8.

Address for correspondence.

Salvador Carrasco-Sosa

Depto. Ciencias de la Salud, T-172. UAM-I, CDMX, México. scas@xanum.uam.mx 